\title{
Factors affecting the proliferation of cardiomyocyte
}

\author{
Xixian $\mathrm{Cao}^{1}$ \\ $+8618054103669$ \\ ${ }^{1}$ Jiu Quan middle school, Gan Su China
}

\begin{abstract}
Heart is one of the most significant organs in mammalian animals, it functions as a pump to make the blood flow from heart to the body tissue and turn back to heart, which can provide the oxygen and other nutrients with the body tissue and carry the waste from the body 86tissue. There is an increase found in the incidence of heart disease and the case fatality rate of heart disease all over the world, this is because the heart disease leads to the greater amounts of cardiomyocyte dead and the capability of cardiomyocyte proliferation is weaker. To treat the heart disease and recover the capability of cardiomyocyte proliferation, this article summaries three factors that can affect the capability of cardiomyocyte proliferation, which may help with the treatment of heart disease in the future.
\end{abstract}

\section{INTRODUCTION}

One of the most significant organs in mammals is heart[13]. With the development of new technology, there are some investigations about the factors which have an influence on the proliferation of cardiomyocytes[4-6]. There are four cavities in the heart, which are ventricles and atriums. In addition, the arteries connect with the ventricles, and the veins always connect with the atriums. With the help of the special muscle---cardia muscle, the heart will contract and release regulatory working as a pump to make the blood flow to the body tissue and turn back to the heart. Meanwhile, there are the valves between the same side of ventricle and atrium, but the different side of ventricle and atrium will not connect with each other, which can control the flowing direction of blood. In the microcosm, the heart consists of three specific types of cell: cardiomyocytes, fibroblasts, and non-striated muscle. With the contracting and releasing of cardia muscle, the blood will flow from the heart to the body tissue and turn back to the heart, which is called double circulatory system. With the help of the double circulatory system, the blood will provide the oxygen and other nutrients with the body tissue and help to transport the products by metabolize reaction to keep the cells a balanced metabolic. The heart can also keep a balanced temperature [7-9].

The cardiomyocytes are short, branched cells, because the capability of cardiomyocytes proliferation in adult mammals is weak and the process of mitosis in telophase is not successful, there are less one nucleus in a cardiomyocyte. In addition, the cardiomyocytes will combine with each other by intercalated disc. There are few myofibrils in the cardiomyocyte, which can form some regulate bands. By using the different concentration gradient of calcium ions between inside and outside of cardiomyocytes, it can make the myofibrils move to each other to keep the cardio muscle contract and release regulatory [10-12].

The different capability of the cardiomyocyte proliferation in different species. For the non-mammalian animals, there is a growing initiative to determine whether existing cardiomyocytes or progenitor cells can be coaxed into eliciting a regenerative response[13, 14]. For example, using the adult zebrafish in which the cardiomyocytes had been genetically labelled $48 \mathrm{~h}$ after fertilization then, remove the $20 \%$ ventricle and harvest the tissue, then assess the cardiac regeneration at 30 days, then, after 30 days, test the GFP-positive cardiomyocyte in the missing area. After that experiment, the missing area of adult zebrafish is almost complete regenerated. And the function of that area will be recovered, which can prove that the cardiomyocytes are able to proliferate, and the new cardiomyocytes are made by the previous cardiomyocyte's division[15]. But for the mammalian animals, there are some situations about the cardiomyocyte's proliferation Use the one-birth mouse and resect its $10 \%$ apex. After few days, harvest the tissue and obverse the figure of EF, FS. Until 21 days after the experiment, the missing apex will be regenerated, and the function of the area will be almost complete recovered, which means the cardiomyocytes are able to proliferate and the new cardiomyocytes are made by the previous cardiomyocyte's division. But when the seven-day mice are used in the same experiment, after 21 days, the missing apex cannot be total regenerated, which can prove that the capability of cardiomyocytes proliferation are progressively weak[16]. For the pigs, unless one-day birth pigs' cardiomyocytes, another period of pigs' cardiomyocytes are not able to proliferate[17, 18]. For human beings, in the twenty years after birth, their cardiomyocytes have a low level of proliferation [1, 19].

Since the cardiomyocytes of adult mammals cannot 
proliferate, when some cardiomyocytes dead, the missing area are not regenerated, which leading to the adjacent cardiomyocytes hypertrophy to fill the missing area. When there are plenty of cardiomyocytes have been died, the fibroblast will be filled with the missing area to form the cicatrix tissue, which can cause that the heart cannot contract and release in a normal way. So how to improve the ability of cardiomyocyte proliferation is the most important stage of treating the heart disease. In this article, we will investigate the different factors that can have the influence on the cardiomyocyte proliferation, which may be helpful to the treatment of heart disease.

\section{DIFFERENT FACTORS IN CARDIOMYOCYTE PROLIFERATION}

\subsection{Nerves}

Used the adult zebrafish and make an operation to resect its apex, 30 days after the operation, the missing area will be regenerated, there were plenty of nerves forming at the same time. Then, used the same experiment to the adult zebrafish whose nerves were reduced by transgenic which can diminish the cardiomyocyte cell-cycle activity and inhibited heart regeneration following the operation. After 30 days, there were numerous collagens forming in the injured area, the capability of cardiomyocyte proliferation will be weaker than ever before, which can prove that when the heart of the adult zebrafish regenerate, there is a number of the nerves forming at the same time.

If the growth of the nerve is controlled in this period, it can not only have an influence on the capability of cardiomyocyte proliferation, but also affect the regeneration of heart. To get the more accurate result about the effect of the nerves in the cardiomyocyte proliferation, the investigator did the other experiment about just born mouse. Firstly, resected postnatal day 1 mouse apex, then inject the medicine (carbachol, which can inhibit nerves' function) to the mouse, after seven days, the $\mathrm{pH} 3+$ and Aurora $\mathrm{B}+$ cardiomyocytes decreased compared with the normal mice, which may indicate that the capability of the cardiomyocyte proliferation decreased.

When the investigator does an operation that cannot only excise the nerve fragment in the carotid artery of the vague nerve, but also resect the apex of the mice. Seven days after that operation, tested the level of ph3+ and Aurora B, the capability of cardiomyocyte proliferation will be weaker than the normal mice.

Through the above three experiments, the researchers used transgenic, physical damage and drug effects to inhibit the growth of neuronal cells, and explored whether neuronal cells have an effect on the proliferation of cardiac myocytes. The experiment proved that when inhibiting the growth of neuronal cells, the proliferation capacity of cardiac myocardial cells is significantly reduced, and neuronal cells have an important effect on the proliferation of cardiac myocardial cells (Figure 1)[20, $21]$.

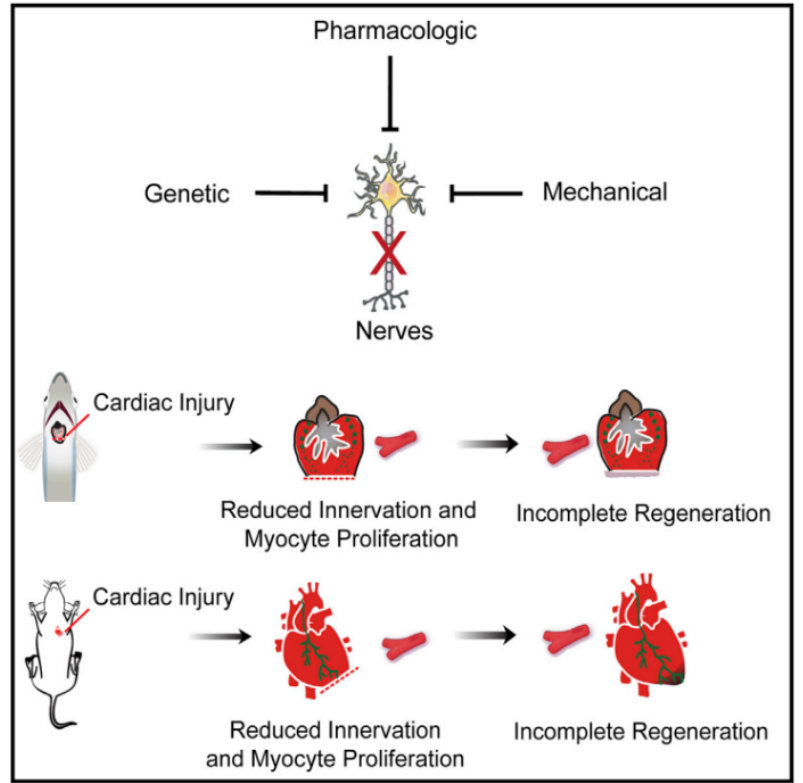

Fig1. Scheme of nerves regulate cardiomyocyte proliferation and heart regeneration.

\subsection{MicroRNA}

\subsubsection{MiR-17-92}

The length of the microRNA is only as long as fifteen to twenty bases and the microRNA cannot translate to the protein, which can bind to the mRNA to change the gene expression [6, 22]. By the research, there is a high level of miR-17-92 when the mouse grows up, and this type of RNA can improve the other type of the cell division. So, there is a hypothesis about whether the miR-17-92 can promote the capability of the cardiomyocyte proliferation. By using the transgenic method, we can knockdown the miR-17-92. The volume of the heart decrease in both the homozygous and heterozygous of the mice, especially the volume of heart of the homozygous are the smallest. Then measure the mean cardiomyocyte area, they found the transgenic mice is larger than others and by detecting the heart to body ratio ( $\mathrm{HW} / \mathrm{BW}$ ), the heart function of transgenic mice is the same compared with the normal. Above results can indicated that decrease the mir-17-92 may reduce the total number of the cardiomyocyte.

To test this hypothesis, the detection of $\mathrm{PH} 3$ value proved that inhibition of miR-17-92 significantly reduced the proliferation ability of mouse cardiac myocytes. Through transgenic methods, miR-17-92 was inserted into the heart muscle cells of the mouse heart two months after birth, and the volume of the mouse heart was measured. The volume of the heart of the transgenic mouse was found to be larger, indicating that miR-17-92 may have a promoting effect in the cardiomyocytes of mice heart. The detection of $\mathrm{PH} 3$ value proved that miR17-92 did had an effect on the proliferation ability of mouse cardiac myocytes.

If the mouse myocardial cells overexpress the miR-1792 at the age of 6 months, and MI cardiac surgery is performed, it is found that the collagen in the mouse heart gradually disappears, proving the ability of proliferation 
of the mouse cardiomyocytes has been enhanced, miR-1792 promotes cardiac regeneration in mice [23].

\subsubsection{MiR-15}

The study found that during the development and growth of mice, the expression level of the miR-15 family of mouse heart cardiomyocytes increased, and with the growth and development of mice, the proliferation ability of mouse heart cardiomyocytes decreased. It is inferred that the miR-15 family may have an inhibitory effect on the proliferation of cardiac myocytes. In order to test this hypothesis, the overexpression of miR-15 family genes in embryonic mouse cardiac myocytes revealed that the size of the mouse heart became smaller, the number of cardiac muscle cells decreased, and the number of genes that inhibited cardiac myocytes increased, the ratio of binuclear myocardial cells increased. In addition, the miR-15 family in mouse heart myocardial cells after birth was inhibited. Through the detection of $\mathrm{PH} 3$ value, it was found that the proliferation ability of mouse heart myocardial cells was increased, and the number of genes that promote the proliferation of mouse heart myocardial cells increased. The above experiments prove that the miR-15 family has an inhibitory effect on the proliferation of cardiac myocytes (Figure 2)[24].

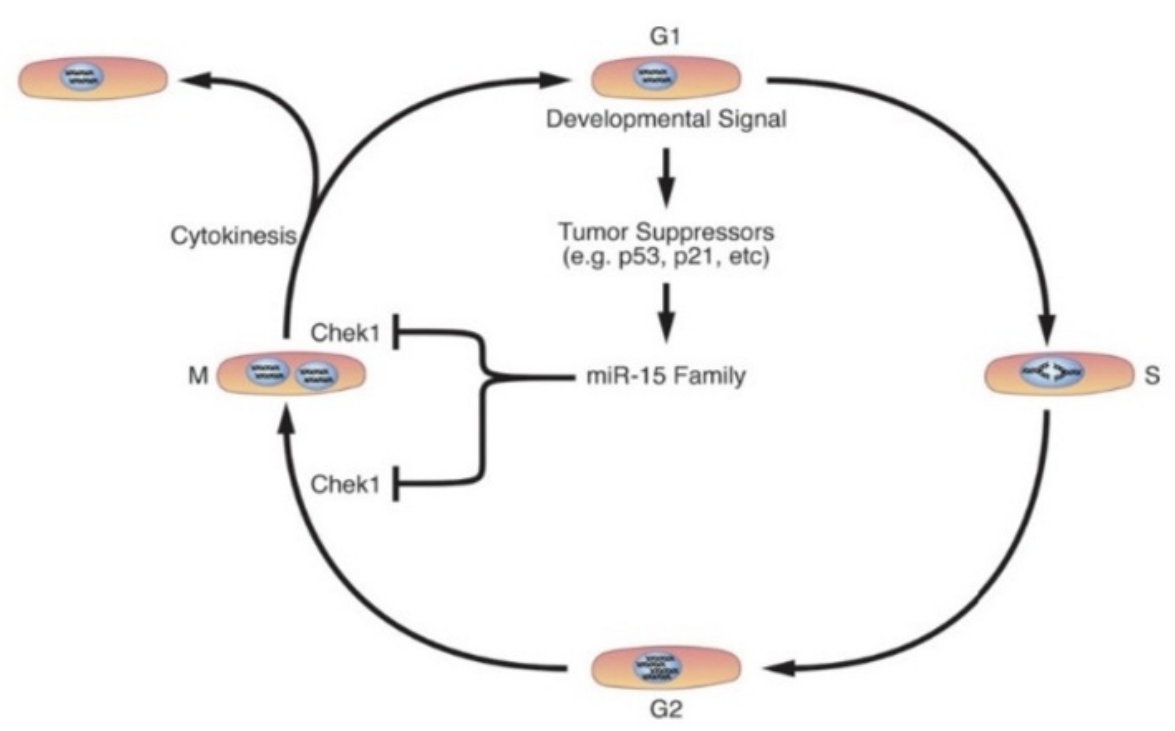

Fig2. Scheme of miR-15 regulate cardiomyocyte proliferation and heart regeneration.

\subsection{Extracellular matrix-periostin}

Periostin is the main component of the extracellular matrix, and studies have shown that the expression of periostin has increased during the early development of the heart. When the heart, blood vessels, and skeletal muscle are damaged, the expression of periostin will increase. It can be speculated that the periosin may promote the proliferation of cardiac myocytes.

To test this hypothesis, Cardiomyocytes were cultured in an in vitro culture dish and periostin was added. After a period of time, the number of cardiomyocytes was found to be increased, which indicates that periostin can promote the proliferation of cardiomyocytes. Periostin was injected into the heart through surgery in vivo, and the function and weight of the heart were tested through control experiments, and it was found that the surgical injury had no effect on the function of the heart. After a period of time, the detection of $\mathrm{PH} 3$ and Aurora B value found that the number of cardiac myocytes increased and the ratio of mononuclear cells increased, proving that periostin can promote the proliferation of cardiac myocytes.

To further confirm this hypothesis, Periostin was injected at the same time when the heart was undergoing MI surgery. After a period of time, it was found that the volume of the heart gradually decreased. The detection of PH3 and Aurora B values showed that the proliferative capacity of cardiac myocytes increased, and it was found that the ratio of collagen in the heart at this time gradually decreases, thus proving that periostin can promote the proliferation of cardiac myocytes and make the damaged heart have the ability to regenerate[25, 26].

\section{DISCUSSION}

An increase is found in the in the incidence of heart disease and the case fatality rate of heart disease all over the world[3], since the heart disease can cause the cardiomyocyte dead and the capability of cardiomyocyte proliferation is weaker, so how to improve the capability of cardiomyocyte proliferation is the most significant step in the treatment and recovery of heart disease.

In this article, there are three impacts which can affect the capability of cardiomyocyte proliferation (the nerve, the microRNA, the extracellular matrix of periostin). The nerve can improve the capability of cardiomyocyte proliferation. When the growth of nerve is inhibited, the capability of cardiomyocyte proliferation will diminish and the regeneration of heart also keeps a downward trend. Different type of microRNA has different impacts about the capability of cardiomyocyte proliferation. For 
example, the miR-17-92 can strengthen the capability of cardiomyocyte proliferation. When the expression of miR-17-92 is exceeded, the capability of cardiomyocyte proliferation can be improved. So, the regeneration of heart will keep an upward trend. By contrast, the miR-15 family can repress the capability of cardiomyocyte proliferation. When the expression of miR-15 family is exceeded, the capability of cardiomyocyte proliferation will be suppressed and the regeneration of heart will be weaker than that of normal one. In addition, the extracellular matrix can also affect the capability of cardiomyocyte proliferation. For example, when the content of periostin is exceeded, the capability of cardiomyocyte proliferation will be promoted. So, the regeneration of heart will be advanced as well.

These experiments of impacts which have the influence on the capability of cardiomyocytes proliferation are the animal tests, which can only provide some suggestions for the treatment of human heart disease. It has not been proving that the same influence during these experiments affects human beings. As a result, there are more experiments being finished in the near future, which can totally confirm that whether these impacts can affect the capability of cardiomyocyte proliferation about human beings. Actually, these experiments only guide the new methods about the treatment of heart disease. But it should improve the targetability of drugs and avoid the side effects of these drugs. To study medicine that may contain microRNA expression, these medicines should be used carefully to avert the less targetability of medicine by gene mutation and improve the security of medicine. This article may provide some suggestions for the treatment of heart disease.

\section{REFERENCE}

1. Marcello, et al., Human heart failure: Is cell therapy a valid option? Biochemical Pharmacology, 2014.

2. Shirakabe, A., et al., Aging and Autophagy in the Heart. Circ Res, 2016. 118(10): p. 1563-76.

3. Linton, P.J., et al., This old heart: Cardiac aging and autophagy. J Mol Cell Cardiol, 2015. 83: p. 44-54.

4. Bersell, K., et al., Neuregulin1/ErbB4 Signaling Induces Cardiomyocyte Proliferation and Repair of Heart Injury. Cell, 2009. 138(2): p. 257-270.

5. Mahmoud, A.I., et al., Meis1 regulates postnatal cardiomyocyte cell cycle arrest. Nature, 2013. 497(7448): p. 249-253.

6. Tian, Y., et al., A microRNA-Hippo pathway that promotes cardiomyocyte proliferation and cardiac regeneration in mice. Sci Transl Med, 2015. 7(279): p. $279 \mathrm{ra3} 8$.

7. Stanley, W.C., F.A. Recchia, and G.D. Lopaschuk, Myocardial substrate metabolism in the normal and failing heart. Physiol Rev, 2005. 85(3): p. 1093-129.

8. James, T.N., Structure and function of the sinus node, AV node and his bundle of the human heart: part II-function. Prog Cardiovasc Dis, 2003. 45(4): p. $327-$ 60 .
9. Sedmera, D., Function and form in the developing cardiovascular system. Cardiovasc Res, 2011. 91(2): p. 252-9.

10. Schirone, L., et al., A Review of the Molecular Mechanisms Underlying the Development and Progression of Cardiac Remodeling. Oxid Med Cell Longev, 2017. 2017: p. 3920195.

11. Prabhu, S.D. and N.G. Frangogiannis, The Biological Basis for Cardiac Repair After Myocardial Infarction: From Inflammation to Fibrosis. Circ Res, 2016. 119(1): p. 91-112.

12. Später, D., et al., How to make a cardiomyocyte. Development, 2014. 141(23): p. 4418-31.

13. Uygur, A. and R.T. Lee, Mechanisms of Cardiac Regeneration. Developmental Cell, 2016. 36(4): p. 362-374.

14. Polizzotti, B.D., et al., A cryoinjury model in neonatal mice for cardiac translational and regeneration research. Nature Protocols, 2016. 11(3): p. 542-552.

15. Jopling, C., et al., Zebrafish heart regeneration occurs by cardiomyocyte dedifferentiation and proliferation. Nature, 2010. 464(7288): p. 606-9.

16. Porrello, E.R., et al., Transient Regenerative Potential of the Neonatal Mouse Heart. Science, 2011. 331(6020): p. 1078-1080.

17. Velayutham, N., E.J. Agnew, and K.E. Yutzey, Postnatal Cardiac Development and Regenerative Potential in Large Mammals. Pediatric Cardiology, 2019. 40(7): p. 1345-1358.

18. Ye, L., et al., Early Regenerative Capacity in the Porcine Heart. Circulation, 2018. 138(24): p. 27982808.

19. Bergmann, O., et al., Dynamics of Cell Generation and Turnover in the Human Heart. Cell, 2015. 161(7): p. $1566-1575$.

20. Mahmoud, A.I., et al., Nerves Regulate Cardiomyocyte Proliferation and Heart Regeneration. Dev Cell, 2015. 34(4): p. 387-99.

21. Ieda, M., Heart development and regeneration via cellular interaction and reprogramming. Keio J Med, 2013. 62(4): p. 99-106.

22. Wojciechowska, A., A. Braniewska, and K. KozarKamińska, MicroRNA in cardiovascular biology and disease. Adv Clin Exp Med, 2017. 26(5): p. 865-874.

23. Chen, J., et al., mir-17-92 cluster is required for and sufficient to induce cardiomyocyte proliferation in postnatal and adult hearts. Circ Res, 2013. 112(12): p. 1557-66.

24. Porrello, E.R., et al., MiR-15 family regulates postnatal mitotic arrest of cardiomyocytes. Circ Res, 2011. 109(6): p. 670-9.

25. Hudson, J.E. and E.R. Porrello, Periostin paves the way for neonatal heart regeneration. Cardiovasc Res, 2017. 113(6): p. 556-558. 
26. Shimazaki, M., et al., Periostin is essential for cardiac healing after acute myocardial infarction. J Exp Med, 2008. 205(2): p. 295-303. 